\title{
Ultrasound semiautomatic versus manual estimation of carotid intima-media thickness: reproducibility and cardiovascular risk stratification
}

\author{
Caterina Beatrice Monti', Marco Alì ${ }^{2,5}$, Davide Capra1, Federico Wiedenmann ${ }^{3}$, Giulia Lastella ${ }^{4}$, \\ Francesco Secchi ${ }^{1,5}$, Francesco Sardanelli, ${ }^{1,5}$
}

${ }^{1}$ Department of Biomedical Sciences for Health, Università degli Studi di Milano, ${ }^{2}$ Unit of Diagnostic Imaging and Stereotactic Radiosurgery, Centro Diagnostico Italiano, ${ }^{3}$ Medicine and Surgery School, Università degli Studi di Milano, ${ }^{4}$ Postgraduation School in Radiodiagnostics, Università degli Studi di Milano, ${ }^{5}$ Unit of Radiology, IRCCS Policlinico San Donato, Milan, Italy

\begin{abstract}
Aims: Carotid intima-media thickness (CIMT) is used increasingly as an imaging biomarker of cardiovascular risk (CVR). Our aim was to compare semiautomatic CIMT (sCIMT) versus manual CIMT (mCIMT) for reproducibility and prediction of CVR. Materials and methods: Two independent readers measured sCIMT and MCIMT on previously acquired images of the right common carotid artery of 200 consecutive patients. Measurements were performed twice, four weeks apart; sCIMT was reported along with an image quality index (IQI) provided by the software. CVR stratification was compared for thresholds established by mCIMT studies, adapted for sCIMT according to a regression model. Results: sCIMT (median $0.67 \mathrm{~mm}$, interquartile range [IQR] $0.57-0.76 \mathrm{~mm}$ ) was significantly lower $(\mathrm{p}<0.001)$ than mCIMT (median $0.76 \mathrm{~mm}, \mathrm{IQR} 0.63-0.84$ $\mathrm{mm} ; \rho=0.832, \mathrm{p}<0.001$, slope 0.714 , intercept 0.124 ). Overall, intra-reader reproducibility was $76 \%$ for sCIMT and $83 \%$ for mCIMT $(\mathrm{p}=0.002)$, inter-reader reproducibility $75 \%$ and $76 \%$, respectively $(\mathrm{p}=0.316)$. In 129 cases with IQI $\geq 0.65$, reproducibility was significantly higher $(\mathrm{p} \leq 0.004)$ for sCIMT than for mCIMT (intra-reader $85 \%$ versus $83 \%$, inter-reader $80 \%$ versus $77 \%$,). The agreement between sCIMT and mCIMT for CVR stratification was fair both overall $(\kappa=0.270)$ and for IQI $\geq 0.65$ $(\kappa=0.345)$, crude concordance being $79 \%$ and $88 \%$, respectively. Conclusions: Reproducibility of sCIMT was not higher than mCIMT overall but sCIMT was significantly more reproducible than mCIMT for high-IQI cases. sCIMT cannot be used for CVR stratification due to fair concordance with mCIMT, even for high IQI. More research is required to improve image quality and define sCIMT-based thresholds for stratification of CVR.
\end{abstract}

Keywords: carotid intima-media thickness; Doppler; cardiovascular diseases; reproducibility of results; computer-assisted

\section{Introduction}

Stroke is a major cause of cardiovascular death, representing the second worldwide leading killer after ischemic heart disease [1]. In Europe, stroke has an age-

Received 16.01.2019 Accepted 25.03.2020

Med Ultrason

2020, Vol. 22, No 4, 402-408

Corresponding author: Dr. Davide Capra

Department of Biomedical Sciences for Health

Università degli Studi di Milano

Via Mangiagalli 31, 20133, Milano, Italy

Phone: +39025277 4468

Fax: +3902 52774925

E-mail: davide.capra@unimi.it standardized incidence of 95 to $290 / 100,000$ per year, with a mortality ranging from $15 \%$ to $35 \%$ [2]. Up to now, different scores to assess the risk of cardiovascular events have been developed [3,4], but they are still not completely satisfactory: many clinical events still occur in patients considered to be at low or intermediate risk $[5,6]$.

Carotid atherosclerosis is one of the main risk factors for ischemic stroke [7]. Carotid intima-media thickness (CIMT) represents the width of the innermost portion of the carotid artery wall and may increase as atherosclerosis progresses, due to deposition of lipids and intimal proliferation [8]. Thus, CIMT has emerged as a predictor of cardiovascular events: its measurement represents a class IIa recommendation for cardiovascular risk (CVR) 
assessment, where CVR is defined as a risk for a healthy adult to develop cardiovascular events associated with atherosclerotic vascular disease [9]. The study of Polak et al [10], conducted on the Framingham cohort, found that a significantly higher number of patients with larger CIMT values reported cardiovascular disease at followup when compared to those with normal CIMT values. Nevertheless, the use of CIMT for cardiovascular risk assessment is still debated, since there are no standardized methods for its measurement and no established reference values for normality [11]. The most important technical discrepancies between reported methods of CIMT measurement may be found in the choice of the carotid segment to evaluate and in measurements which are reported, which could be means or maximums or any combination of the two [12].

In clinical practice, CIMT may be measured with a routine ultrasonography of the supra-aortic vessels; therefore, its assessment is easy, low-cost and non-invasive [13]. Semiautomatic methods for CIMT calculation have become increasingly popular in the last years, since they allow quick measurements and a supposedly higher precision, possibly overcoming the limitations of nonstandardized manual evaluation [14].

The purpose of our study was to compare semiautomatic CIMT versus manual CIMT for reproducibility and prediction of cardiovascular risk.

\section{Materials and methods}

The local Ethics Committee approved this study (Ethics Committee of San Raffaele Clinical Research Hospital; protocol code CardioRetro; approved on March 9th, 2017; amended on May 10th, 2018). "This study was partially supported by Ricerca Corrente founding from Italian Ministry of Health to IRCCS Policlinico San Donato. There was no overlap with subjects from previous research performed at our Institution. This research received no specific grant from any funding agency in the public, commercial or not-for-profit sectors. Due to the retrospective design of this study, specific informed consent was waived.

Ultrasound B-mode images of consecutive patients who underwent a color-Doppler ultrasound of the supra-aortic vessels at our institution were retrospectively re-evaluated for CIMT assessment. All patients who underwent color-Doppler ultrasound of the supra-aortic vessels were included in the study, regardless of sex, age, or cardiovascular risk factors. Exclusion criteria were repeated examinations on the same patient, significant carotid stenosis or previous treatments for carotid stenosis.

\section{Image acquisition}

Ultrasound examinations were conducted using a RS80A Prestige (Samsung Healthcare, Seoul, South Korea) equipment with a $7.5-\mathrm{MHz}$ linear transducer with a field of view of $50 \mathrm{~mm}$. Patients were examined while lying supine or sitting down; when that was not an option, with a slightly extended neck. One image per patient of the right common carotid that permitted a good visualization of CIMT was stored.

\section{Image processing}

Average CIMT of the right common carotid artery was assessed twice, 4 weeks apart, by a radiologist with ten years of experience in carotid ultrasound, and once by a second radiologist with two years of experience, with two different methods: semiautomatic and manual. The first set of measurements of the more experienced reader along with the other two sets of measurements were used for the assessment of reproducibility.

A dedicated semiautomatic software (AutoIMT+, Samsung Healthcare, Seoul, South Korea) was utilized for semiautomatic CIMT calculations. When such software failed to identify the common carotid artery, for instance measuring a section of muscle, readers could manually correct it by selecting the center of the desired vessel. AutoIMT + software provided CIMT measurement, the number of points used for CIMT calculation and a so-called "quality index" (QI), related to the ratio between length of the measured segment and number of points, which were both reported along with CIMT.

For manual CIMT calculation, each radiologist took three different measurements, as recommended by the Italian Society for Vascular Investigation $[11,12,15]$ using a dedicated software for image viewing. Such measurements were then averaged.

A comparison between semiautomatic and manual measurements is presented in figure 1 .

\section{Statistical analysis}

Continuous variables were tested for normality with the Shapiro-Wilk test. Parametric data were reported as

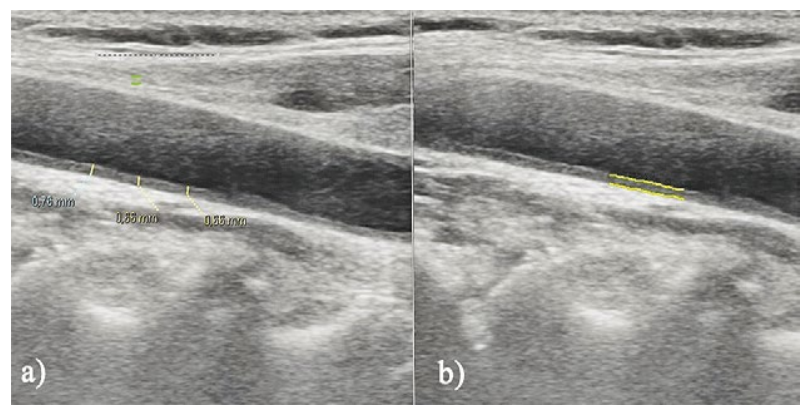

Fig 1. Comparison between semiautomatic and manual carotid intima-media thickness measurements in a 73-year-old female. 
a mean \pm standard deviation (SD), while non-parametric data were reported as a median and interquartile range.

Intra- and inter-reader reproducibility of both semiautomatic and manual CIMT measurements were assessed with Bland-Altman analysis, and it was reported as a complement to one of the ratio between two times the SD and mean measure, along with the coefficient of reproducibility $(\mathrm{CoR})$ and bias. Differences between data reproducibility were assessed with the F-test of equality of variances.

Differences between semiautomatic and manual data were assessed with the $t$-test when variables were normally or near-normally distributed, or by the Wilcoxon test when variables were not normally or near-normally distributed. Correlations between semiautomatic and manual data as well as between image quality and points of semiautomatic measurements were appraised with Pearson or Spearman correlation with regards to data distribution. Correlation coefficients were interpreted according to Evans [16]. Concordance between semiautomatic and manual CIMT with regards to cardiovascular risk stratification were assessed with Cohen $\kappa$ as a measure of agreement, as well as reporting the percentage of crude concordant cases. Thresholds for manual CIMT were chosen according to Lorenz et al [17] and adapted according to a regression model: namely the correlation between semiautomatic and manual CIMT was modelized, and thresholds for semiautomatic CIMT were obtained from the relation between the two. CIMT was considered positive for cardiovascular risk when equal to or above threshold, negative when below.

Subgroup analyses were performed according to the value of QI, using different QI thresholds and calculating both manual and semiautomatic CIMT reproducibility by only including patients whose semiautomatic QIs were equal to or above the desired thresholds. If overcoming one particular QI threshold, semiautomatic CIMT reproducibility went from lower to higher than that of manual CIMT, such threshold was used for further subgroup analysis.

Statistical analysis was performed with SPSS v22 (IBM SPSS Inc., Chicago, IL, USA), and MATLAB R2018b (Mathworks, Natick, MA, USA); $P$-values $<0.05$ were considered statistically significant.

\section{Results}

\section{Overall results}

The color Doppler examinations of supra-aortic vessels of 200 consecutive patients (median 71 years, interquartile range [IQR] 63-78 years), conducted from May 2017 to December 2017 at our institution, were retro-
Table I. Study population characteristics

\begin{tabular}{ll}
\hline Patients characteristics $(\mathbf{n}=\mathbf{2 0 0})$ & \\
\hline Age (years) & $71(63-78)$ \\
Females (n,\%) & $103(51)$ \\
Manual CIMT (mm) & $0.76(0.63-0.84)$ \\
Semiautomatic CIMT (mm) & $0.67(0.57-0.76)$ \\
\hline
\end{tabular}

Data are reported as median and interquartile range. CIMT: carotid intima-media thickness

spectively analyzed. Among these patients, 103 (51\%) were females. The characteristics of the study population are reported in Table I.

The software AutoIMT+ failed to properly detect the common carotid artery on the stored image in $15 \%$ of patients $(\mathrm{N}=30)$. In such cases, manual adjustments were made: another point at the center of the vessel was chosen, so that the detection could succeed.

Semiautomatic CIMT values $(0.67 \mathrm{~mm}, 0.57-0.76$ $\mathrm{mm})$ were significantly lower $(\mathrm{p}<0.001)$ than manual CIMT values $(0.76 \mathrm{~mm}, 0.63-0.84 \mathrm{~mm})$ and a strong positive correlation $(\rho=0.832, p<0.001)$ was found between the two measurements across the entire population. The correlation was modeled into a regression line with a slope of 0.714 and an intercept of 0.124 (fig 2). Such a trendline was then used to translate thresholds for manual CIMT to use them for semiautomatic CIMT. Semiautomatic CIMT detected 48 high-risk patients, while manual CIMT showed 21 patients at high cardiovascular risk.

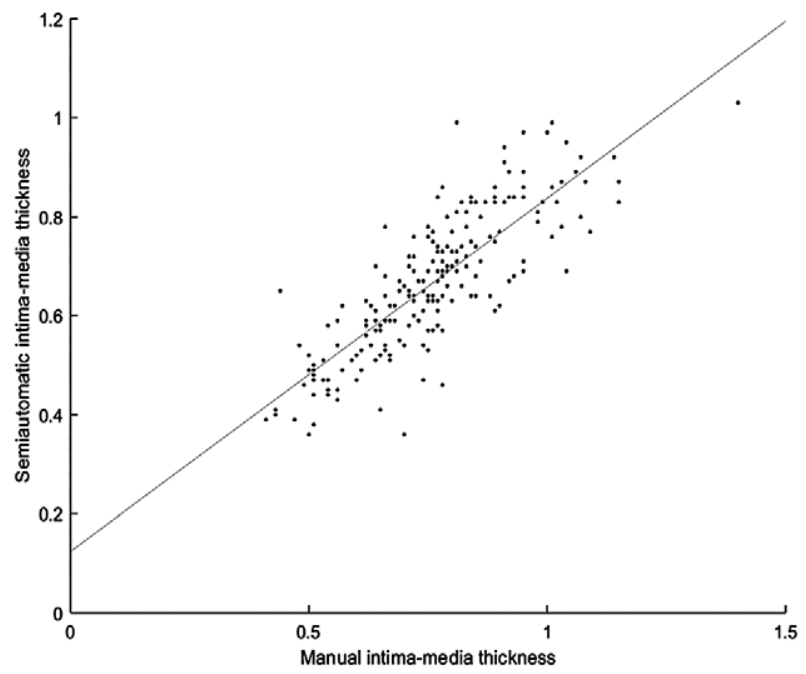

Fig 2. Correlation between semiautomatic and manual carotid intima-media thickness (CIMT), $\rho=0.832, p<0.001$, modeled into a regression line with a slope of 0.714 and an intercept of 0.124 . This regression model was then used for translating thresholds for manual CIMT to use them for semiautomatic CIMT. 
Semiautomatic and manual CIMT agreed on 144 negative cases and 13 positive cases, 35 cases were positive for semiautomatic CIMT and negative for manual CIMT, and 8 vice versa. Agreement for risk prediction was fair $(\kappa=0.270)$, with a crude concordance of $79 \%$.

Intra-reader reproducibility on the whole study sample was $76 \%$ with a bias of $0.003 \mathrm{~mm}$ and CoR of 0.163 $\mathrm{mm}$ for semiautomatic CIMT assessment and $83 \%$ with a bias of $-0.014 \mathrm{~mm}$ and CoR of $0.130 \mathrm{~mm}$ for manual CIMT calculation. Inter-reader reproducibility on the whole study sample was $75 \%$ with a bias of -0.001 $\mathrm{mm}$ and CoR of $0.170 \mathrm{~mm}$ for semiautomatic CIMT assessment and $76 \%$ with a bias of $0.024 \mathrm{~mm}$ and CoR of $0.130 \mathrm{~mm}$ for manual CIMT calculation. Intra-reader reproducibility was significantly higher for manual CIMT ( $p=0.002$ ), while inter-reader reproducibility was
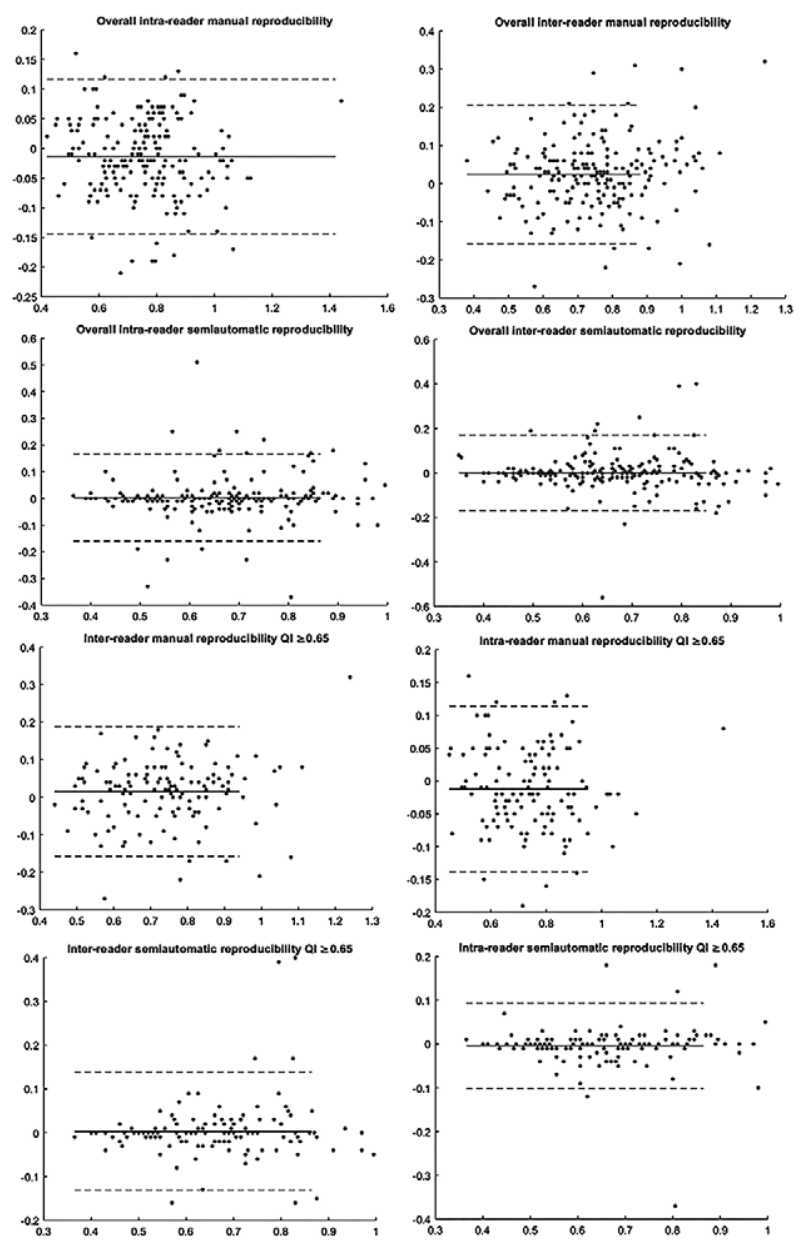

Fig 3. Bland-Altman charts representing the reproducibility of (from top line to the bottom line) manual carotid intima-media thickness (CIMT) over the whole population, overall semiautomatic CIMT, manual CIMT with quality index $\geq 0.65$, and semiautomatic CIMT with quality index $\geq 0.65$. Intra-reader reproducibilities are on the left; inter-reader on the right. not significantly different $(\mathrm{p}=0.316)$ for semiautomatic CIMT than manual CIMT.Bland-Altman plots are shown in figure 3.

A strong positive correlation $(\rho=0.678, \mathrm{p}<0.001)$ was found between QI $(0.86,0.79-1.00)$ and points of length of the section used for measurements (115 points, 81-157 points), between QI and intra-reader reproducibility $(\rho=0.724, p=0.005)$, and between QI and interreader reproducibility $(\rho=0.900, p<0.001)$.

\section{Subgroup analysis}

Results of subgroup analyses for reproducibility according to QI were reported in Table II. The subgroup having a semiautomatic CIMT assessment with $\mathrm{QI} \geq 0.65$ was composed by 129 subjects, 59 males (46\%) and 70 females (54\%), whose median age was 70 years (IQR 61-77 years). There were no significant differences in terms of sex $(\mathrm{p}=0.652)$ or age $(\mathrm{p}=0.882)$ when compared to the entire population. At $\mathrm{QI} \geq 0.65$, intra-reader reproducibility was $85 \%$ with a bias of $-0.004 \mathrm{~mm}$ and CoR of $0.098 \mathrm{~mm}$ for semiautomatic CIMT and $83 \%$ with a bias of $-0.012 \mathrm{~mm}$ and CoR $0.126 \mathrm{~mm}$ for manual CIMT. Inter-reader reproducibility was $80 \%$ with a bias of $0.003 \mathrm{~mm}$ and CoR of $0.013 \mathrm{~mm}$ for semiautomatic CIMT and $77 \%$ with a bias of $0.015 \mathrm{~mm}$ and CoR of $0.172 \mathrm{~mm}$ for manual CIMT. Both intra- and inter-reader

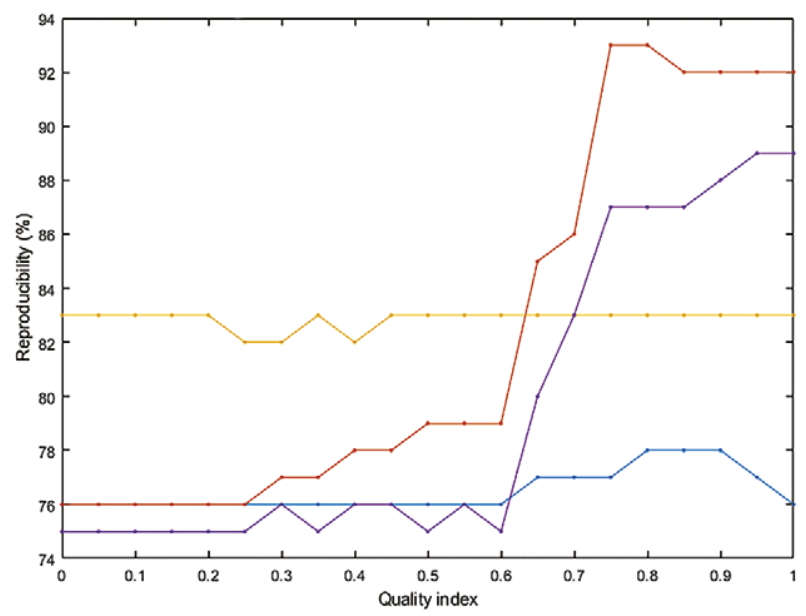

Fig 4. Reproducibility values according to different quality index thresholds. The yellow line represents manual intra-reader reproducibility, the blue line manual inter-reader reproducibility, the purple line semiautomatic inter-reader reproducibility, and the red line semiautomatic intra-reader reproducibility. At a QI threshold $\geq 0.65$, it may be observed that both semiautomatic intra- and inter-reader reproducibility overtake manual intra- and inter-reader reproducibility, respectively (the purple line crossing the blue line, and the red line crossing the yellow line). Moreover, it may be noticed that while manual reproducibility values tend to remain constant with regards to QI, semiautomatic values encounter a steep growth as the QI rises. 
Table II. Reproducibility of measurements of the right carotid intima-media thickness (CIMT) for different quality index (QI) thresholds. Bold data indicates significantly lower reproducibility for semiautomatic CIMT, while italic data indicates significantly higher reproducibility for semiautomatic CIMT. Note that both intra- and inter-reader reproducibility become significantly higher for semiautomatic CIMT than manual CIMT when the QI threshold is $\geq 0.65$.

\begin{tabular}{|c|c|c|c|c|c|c|c|}
\hline \multirow{2}{*}{$\begin{array}{l}\text { QI } \\
\text { threshold }\end{array}$} & \multirow{2}{*}{$\begin{array}{l}\mathrm{N}^{\circ} \text { of } \\
\text { patients }\end{array}$} & \multicolumn{4}{|c|}{ Reproducibility (\%) } & \multicolumn{2}{|c|}{ p-value } \\
\hline & & $\begin{array}{l}\text { Intra-observer } \\
\text { manual }\end{array}$ & $\begin{array}{l}\text { Inter-observer } \\
\text { manual }\end{array}$ & $\begin{array}{l}\text { Intra-observer } \\
\text { semiautomatic }\end{array}$ & $\begin{array}{l}\text { Inter-observer } \\
\text { semiautomatic }\end{array}$ & Intra & Inter \\
\hline 0.00 & 200 & 83 & 76 & 76 & 75 & 0.002 & 0.316 \\
\hline 0.05 & 199 & 83 & 76 & 76 & 75 & 0.003 & 0.252 \\
\hline 0.10 & 198 & 83 & 76 & 76 & 75 & 0.003 & 0.255 \\
\hline 0.15 & 196 & 83 & 76 & 76 & 75 & 0.003 & 0.329 \\
\hline 0.20 & 193 & 83 & 76 & 76 & 75 & 0.006 & 0.386 \\
\hline 0.25 & 188 & 82 & 76 & 76 & 75 & 0.010 & 0.248 \\
\hline 0.30 & 185 & 82 & 76 & 77 & 76 & 0.056 & 0.195 \\
\hline 0.35 & 181 & 83 & 76 & 77 & 75 & 0.030 & 0.325 \\
\hline 0.40 & 174 & 82 & 76 & 78 & 76 & 0.114 & 0.174 \\
\hline 0.45 & 164 & 83 & 76 & 78 & 76 & 0.169 & 0.264 \\
\hline 0.50 & 156 & 83 & 76 & 79 & 75 & 0.374 & 0.314 \\
\hline 0.55 & 148 & 83 & 76 & 79 & 76 & 0.244 & 0.277 \\
\hline 0.60 & 141 & 83 & 76 & 79 & 75 & 0.335 & 0.451 \\
\hline 0.65 & 129 & 83 & 77 & 85 & 80 & 0.004 & 0.006 \\
\hline 0.70 & 122 & 83 & 77 & 86 & 83 & $<0.001$ & $<0.001$ \\
\hline 0.75 & 110 & 83 & 77 & 93 & 87 & $<0.001$ & $<0.001$ \\
\hline 0.80 & 104 & 83 & 78 & 93 & 87 & $<0,001$ & $<0.001$ \\
\hline 0.85 & 95 & 83 & 78 & 92 & 87 & $<0.001$ & $<0.001$ \\
\hline 0.90 & 90 & 83 & 78 & 92 & 88 & $<0.001$ & $<0.001$ \\
\hline 0.95 & 79 & 83 & 77 & 92 & 89 & $<0.001$ & $<0.001$ \\
\hline 1.00 & 64 & 83 & 76 & 92 & 89 & $<0.001$ & $<0.001$ \\
\hline
\end{tabular}

reproducibility of semiautomatic CIMT were significantly higher ( $p=0.004$ and $p=0.006$, respectively) than those of manual CIMT. The correlation between QI and points led to the correspondence of a threshold of 24 points for $\mathrm{QI} \geq 0.65$. A chart depicting the variations of reproducibility values according to QI thresholds may be seen in figure 4.

In this subgroup, while the CIMT semiautomatic values $(0.66 \mathrm{~mm}$, IQR $0.55-0.75 \mathrm{~mm})$ were significantly lower $(\mathrm{p}<0.001)$ than manual CIMT values $(0.75 \mathrm{~mm}$, IQR $0.64-0.83 \mathrm{~mm}$ ), a very strong positive correlation $(\rho=0.867, \quad p<0.001)$ persisted between semiautomatic and manual CIMT assessment. Semiautomatic CIMT detected 17 high-risk patients, while manual CIMT showed 8 patients at a high cardiovascular risk. Semiautomatic and manual CIMT agreed on 109 negative cases and 5 positive cases, while 12 cases were positive according to semiautomatic CIMT and negative according to manual CIMT, and 3 vice versa. Agreement for risk prediction between the two measurements was fair $(\kappa=0.345)$, with a crude concordance of $88 \%$.

\section{Discussion}

The aim of our study was to compare semiautomatic CIMT versus manual CIMT for reproducibility and prediction of cardiovascular risk. Overall, semiautomatic CIMT resulted in being not more reproducible than by manual CIMT. However, in the cases with high image quality, reproducibility of semiautomatic CIMT was significantly higher for that of manual CIMT. In addition, the agreement between semiautomatic CIMT and manual CIMT for cardiovascular risk (CVR) stratification was fair both overall and for cases with a high image quality.

Our results should be extendable to the whole Caucasian target population. In fact, the median age of our entire population reflects well that of those at a high risk of stroke [2] and manual CIMT values from our population are compatible with their age [18].

We showed a high precision of manual CIMT: its overall reproducibility is highly satisfactory (intra-reader $83 \%$, inter-reader $81 \%$ ). Thus, we confirm the results by Plasencia Martinez JM, et al. [19] who found a good 
inter-reader reproducibility (intraclass correlation coefficient $79 \%$ ), and a good inter-reader reproducibility (intraclass correlation coefficient $72 \%$ ). In addition, on our whole population, manual CIMT calculation seemed to be more precise than semiautomatic CIMT.

The significant positive correlation between semiautomatic and manual CIMT measurements on our entire population hints that both values are related to the actual value of CIMT. However, the agreement between the two measurements in attributing CVR according to currently used thresholds, derived from manual measurements [17], is only fair. Even with a crude concordance of $79 \%$ between semiautomatic and manual CIMT measurements in the attribution of CVR, agreement according to Cohen $\kappa$ was low $(\kappa=0.270)$, due to an unbalanced distribution of positive and negative cases (the higher number of negative cases compared to positive cases has a high impact on the calculation of $\kappa$ ). This might possibly be caused by the fact that manual CIMT calculation relies on three different measurements taken at different points, while automatic or semiautomatic CIMT measurements use a higher number of points on a whole section of the carotid artery. Therefore, manual averaging may overestimate the length of carotid portions with increased CIMT, since more remarkable points with a higher CIMT tend to be chosen by readers, and thus it may provide a less reliable estimate. Given that the thresholds for CVR derived from manual measurements, semiautomatic measurements may yield a low agreement with manual measurements for risk prediction.

Our positive correlation between QI and length of the analyzed carotid segment can be explained taking into account that an average measure calculated on a greater number of data provides a more reliable estimate. The significant positive correlation between reproducibility and QI suggested that CIMT values obtained on images with a higher QI could be more precise and reproducible. In fact, at a subgroup analysis of cases with a $\mathrm{QI} \geq 0.65$, neither age nor sex had different distributions compared to the entire population, meaning that the higher reproducibility in the chosen subgroup was likely not attributable to differences in such characteristics. Both intra- and inter-reader reproducibility of the semiautomatic method on the subgroup with $\mathrm{QI} \geq 0.65$ were increased to the point of being significantly higher than those of the manual method on the same subgroup. Therefore, semiautomatic CIMT quantification could be more precise than manual estimates, provided that more than 24 points are utilized.

Despite the increase in reproducibility observed using the semiautomatic method taking QI into account, compared to manual CIMT, agreement for risk prediction between semiautomatic and manual CIMT was still low also in the subgroup with $\mathrm{QI} \geq 0.65(\kappa=0.345)$, thus these two methods do not appear interchangeable for CVR prediction, also in the presence of high QI. However, we must consider that there are no reference thresholds for semiautomatic CIMT for CVR assessment, the only available thresholds being derived from manual CIMT measurements [17,18].

This study has limitations, the first being its retrospective monocentric design which means that results are only extendable to a population similar to the one referring to our center. Second, we used a series of images already acquired and stored in our database. This means that an important source of variability (that due to the repetition of the examination) has been taken out of the study design to avoid a confounding factor but this implies that we do not know what happens when the examination is repeated using manual or semiautomatic CIMT measurements. Third, due to the advanced age of some patients, at times the range of motion of their neck was not ideal and did not allow for the best possible CIMT visualization. However, the target age for the use of CIMT as a risk assessment is comparable to that of our population, therefore we expect this limitation to be present in all studies and in clinical practice. Another limitation may be represented by the fact that we appraised CIMT on the right common carotid artery due to it being common practice and for the benefit of continuity. Nevertheless, this approach was already suggested by a previous work by Rosvall et al [20], who also noted that a study by Lorentz et al [21] reported comparable prognostic significance.

In conclusion, using a series of carotid ultrasound images already acquired in a consecutive series of patients, we showed that CIMT measurement using semiautomatic method (AutoIMT + ) has a significantly higher reproducibility than values obtained with manual methods only when considering images with $\mathrm{QI} \geq 0.65$. In addition, due to low concordance, semiautomatic CIMT assessment with AutoIMT+ cannot substitute for manual calculations in the attribution of cardiovascular risk using a threshold derived from studies which used manual CIMT estimate. To exploit the role of semiautomatic CIMT, prospective studies should be conducted assessing the predictive value of semiautomatic CIMT measurement, i.e. using thresholds obtained with semiautomatic measurement.

\section{Conflict of interest: none}

\section{References}

1. GBD 2016 Causes of Death Collaborators.Global, regional, and national age-sex specific mortality for 264 causes of death, 1980-2016: a systematic analysis for the Global 
Burden of Disease Study 2016. Lancet 2017;390:11511210.

2. Béjot Y, Bailly H, Durier J, Giroud M. Epidemiology of stroke in Europe and trends for the 21st century. Presse Med 2016;45:e391-e398.

3. Sayin MR, Cetiner MA, Karabag T, et al. Framingham risk score and severity of coronary artery disease. Herz 2014;39:638-643.

4. Greve SV, Blicher MK, Sehestedt T, et al. Effective risk stratification in patients with moderate cardiovascular risk using albuminuria and atherosclerotic plaques in the carotid arteries. J Hypertens 2015;33:1563-1570.

5. Arnett DK, Blumenthal RS, Albert MA, et al. 2019 ACC/ AHA Guideline on the Primary Prevention of Cardiovascular Disease: A Report of the American College of Cardiology/American Heart Association Task Force on Clinical Practice Guidelines. Circulation 2019;140:e596-e646.

6. Uthoff H, Staub D, Socrates T, et al. PROCAM-, FRAMINGHAM-, SCORE- and SMART-risk score for predicting cardiovascular morbidity and mortality in patients with overt atherosclerosis. Vasa 2010;39:325-333.

7. Hankey GJ. Stroke. Lancet 2017;389:641-654.

8. Baber U, Mehran R, Sartori S, et al. Prevalence, impact, and predictive value of detecting subclinical coronary and carotid atherosclerosis in asymptomatic adults: the BioImage study. J Am Coll Cardiol 2015;65:1065-1074.

9. Greenland P, Alpert JS, Beller GA, et al. 2010 ACCF/AHA guideline for assessment of cardiovascular risk in asymptomatic adults: executive summary: a report of the American College of Cardiology Foundation/American Heart Association task force on practice guidelines. Circulation 2010;122:2748-2764.

10. Polak JF, Pencina MJ, Pencina KM, O’Donnel CJ, Wolf PA, D'Agostino RB Sr. Carotid-wall intima-media thickness and cardiovascular events. N Engl J Med 2011;365:213-221.

11. Ravani A, Werba JP, Frigerio B, et al. Assessment and relevance of carotid intima-media thickness (C-IMT) in pri- mary and secondary cardiovascular prevention. Curr Pharm Des 2015;21:1164-1171.

12. Naqvi TZ, Lee MS. Carotid intima-media thickness and plaque in cardiovascular risk assessment. JACC Cardiovasc Imaging 2014; 7:1025-1038.

13. Nezu T, Hosomi N, Aoki S, Matsumoto M. Carotid intimamedia thickness for atherosclerosis. J Atheroscler Thromb 2016;23:18-31.

14. Centurión OA. Carotid intima-media thickness as a cardiovascular risk factor and imaging pathway of atherosclerosis. Crit Pathw Cardiol 2016;15:152-160.

15. Antignani PL, Benedetti-Valentini F, Aluigi L, et al. Diagnosis of vascular diseases. Ultrasound investigationsguidelines. Int Angiol 2012;31:1-77.

16. Evans J. Straightforward statistics for behavioral sciences. Brooks/Cole Publishing, Pacific Grove, California, 1996.

17. Lorenz MW, von Kegler S, Steinmetz H, Markus HS, Sitzer M. Carotid intima-media thickening indicates a higher vascular risk across a wide age range: prospective data from the Carotid Atherosclerosis Progression Study (CAPS). Stroke 2006;37:87-92.

18. Näslund U, Lundgren A, Vanoli D, Norberg M. Is intimamedia thickness a predictor for cardiovascular risk? - Authors' reply. Lancet 2019;394:381.

19. Plasencia Martínez JM, Garcia Santos JM, Paredes Martinez ML, Pastor AM. Carotid intima-media thickness and hemodynamic parameters: reproducibility of manual measurements with Doppler ultrasound. Med Ultrason 2015;17:167-174.

20. Rosvall M, Persson M, Östling G, et al. Risk factors for the progression of carotid intima-media thickness over a 16year follow-up period: The Malmö Diet and Cancer Study. Atherosclerosis 2015;239:615-621.

21. Lorenz MW, Markus HS, Bots ML, Rosvall M, Sitzer M. Prediction of Clinical Cardiovascular Events With Carotid Intima-Media Thickness. Circulation 2007;115:459-467. 